\title{
Short-term rotational variability of eight KBOs from Sierra Nevada Observatory
}

\author{
J. L. Ortiz, P. J. Gutiérrez, P. Santos-Sanz, V. Casanova, and A. Sota
}

Instituto de Astrofísica de Andalucía, CSIC, Apt 3004, 18080 Granada, Spain

e-mail: ortiz@iaa.es

Received 3 June 2005 / Accepted 29 September 2005

\section{ABSTRACT}

In 2001, we started a CCD photometry programme to study the short-term variability of some of the brightest TNOs and Centaurs from the Sierra Nevada observatory. In this paper, we report our latest results on short-term rotational variability of 7 trans-neptunian objects: Orcus (2004 DW), $2002 \mathrm{AW}_{197}, 2003 \mathrm{AZ}_{84}, 2003 \mathrm{VS}_{2}, 2002 \mathrm{VE}_{95}, 2001 \mathrm{YH}_{140}, 1996 \mathrm{TL}_{66}$, and a Centaur: $2003 \mathrm{CO}_{1}$. Analysis of the photometric data revealed confident periodicities for 6 objects, with all the lightcurve amplitudes smaller than $0.2 \mathrm{mag}$, except for $2003 \mathrm{VS}_{2}$. Considering all the objects for which reliable lightcurve amplitudes have been reported in the literature (32), the new statistics reveal that $31 \%$ of the bodies show variability above $0.15 \mathrm{mag}$, but only $16 \%$ of them display larger amplitudes than 0.4 mag. Here we present a summary of the main results obtained for these objects, and discuss the implications for their basic physical properties.

Key words. minor planets, asteroids - Kuiper Belt - comets: general

\section{Introduction}

Trans-Neptunian objects (TNOs) are cold, minor bodies of the outer Solar System thought to be composed of ice and rock and formed in its early ages. They are the most likely source for the Centaurs and the Short Period Comets, groups which seem to share the same origin in the Kuiper or Edgeworth-Kuiper Belt (e.g. Fernández 1980; Duncan et al. 1988, etc.). It is currently believed that these objects are among the least evolved in the Solar System, so that a study of these bodies would be an important tool for understanding the formation and early evolution of the Solar System.

Current observational studies of TNOs are mainly focused on color photometry or spectroscopy (e.g. Jewitt \& Luu 2001; Boehnhardt et al. 2002; Barucci et al. 2002; Hainaut \& Delsanti 2002; Doressoundiram et al. 2002, 2005; Delsanti et al. 2004; Peixinho et al. 2004; Fornasier et al. 2004; etc.). Some of these studies use statistical analysis to try to find likely correlations between color trends and orbital parameters. Different dynamical families within the TNOs seem to have peculiar color properties which may indicate different evolutionary paths for the different families. Spectroscopic studies are especially suitable for obtaining surface composition and surface properties (or at least constraints on them) provided that the albedos are known.

Some other observational works on TNOs are focused on their short-term variability (e.g. Davies et al. 1998; Hainaut et al. 2000; Gutiérrez et al. 2001; Farnham 2001; Ortiz et al. 2002; Sheppard \& Jewitt 2002; Bauer et al. 2002; Sekiguchi et al. 2002; Peixinho et al. 2002; Ortiz et al. 2003a,b, etc.). These studies can give us hints of the shape, density, and other basic physical properties of these bodies (e.g. Sheppard \& Jewitt 2002), as well as of the original size-shape distribution (Lacerda et al. 2003). Some of these studies can even identify contact binaries (Sheppard \& Jewitt 2004).

Correlation of short-term variability parameters with orbital parameters or searches for trends within Plutinos, Clasical Belt, and SDO families will eventually be possible, when a large enough sample of TNOs has been studied, which is not the case yet. Besides, photometric variability studies of the largest members of the Kuiper Belt are necessary in order to determine rotation periods, which are used in the thermophysical models that ultimately provide the albedo and object diameter. Accurate rotation periods are also needed to study the opposition effect on TNOs (e.g. Belskaya et al. 2003), and they can provide the link needed to check surface heterogeneity as suggested by spectroscopy data (Barucci et al. 2002; Lazzarin et al. 2003, etc.).

Unfortunately, time-resolved photometry requires considerable observing time for each TNO, which is hard to carry out due to the difficulty of getting long observing runs at large telescopes. Smaller telescopes can be used, but only the brightest TNOs and Centaurs can be then targeted.

In 2001, we started a CCD photometry programme to study the short-term variability of some of the brightest TNOs and Centaurs from Sierra Nevada Observatory. Previous papers (Ortiz et al. 2002, 2003a,b, 2004) have reported results from this ongoing programme for 8 TNOs and 6 Centaurs. This paper reports the continuation of the project, increasing the total number of objects considerably. The main results for the new 
Table 1. Dates and geometric data (range) of the objects observed.

\begin{tabular}{|c|c|c|c|c|c|c|}
\hline & Object & $\overline{r_{\mathrm{h}}(\mathrm{AU})}$ & $\overline{\Delta(\mathrm{AU})}$ & $\overline{\alpha \alpha(\operatorname{deg})}$ & No. of images & Observing dates \\
\hline \multicolumn{7}{|l|}{ TNOs } \\
\hline & 2004 DW & 47.6 & $46.74-47.34$ & $0.57-1.17$ & 316 & 8-11, Mar.; 22, 23, 25-27, Apr. (2004) \\
\hline & $2002 \mathrm{AW}_{197}$ & $47.3-47.1$ & $46.19-46.92$ & $0.26-1.13$ & 549 & $\begin{array}{r}29, \text { Nov.; } 1,3,5,7,8, \text { Dec. }(2002) \\
1-3, \text { Feb. }(2003) \\
21-23,25,26, \text { Jan. }\end{array}$ \\
\hline & $2003 \mathrm{AZ}_{84}$ & 45.8 & $44.85-44.89$ & $0.29-0.54$ & 143 & $20,22,25,26$, Jan.; $15-20$, Dec. $(2004)$ \\
\hline & $2003 \mathrm{VS}_{2}$ & 36.4 & $35.65-36.06$ & $0.95-1.44$ & 191 & $\begin{array}{r}22,23,26,28,29, \text { Dec. }(2003) \\
21,22, \text { Jan. }(2004)\end{array}$ \\
\hline & $2002 \mathrm{VE}_{95}$ & 28.0 & $27.06-27.25$ & $0.68-1.18$ & 146 & $\begin{array}{r}29 \text {, Nov., 3, 4, 8, Dec. (2002) } \\
\text { 15-20, Dec. (2004) }\end{array}$ \\
\hline & $2001 \mathrm{YH}_{140}$ & 36.4 & $35.58-35.54$ & $0.65-0.77$ & 37 & 15-20, Dec. (2004) \\
\hline & $1996 \mathrm{TL}_{66}$ & 35.1 & $34.32-34.38$ & $0.90-1.0$ & 67 & 14-19, Dec. (2004) \\
\hline \multicolumn{7}{|l|}{ Centaurs } \\
\hline & $2003 \mathrm{CO}_{1}$ & $11.44-11.35$ & $10.69-10.76$ & $3.55-4.23$ & 307 & 23, 25, 26, Jan.; 19, 20, 23-26, Apr. (2004) \\
\hline
\end{tabular}

objects and the implications for some physical properties are also discussed in this paper.

\section{Observations and data reduction}

The observations were carried out on the Instituto de Astrofísica de Andalucía $1.5 \mathrm{~m}$ telescope at Sierra Nevada Observatory, in Granada, Spain. The bodies observed are listed in Table 1 along with other relevant data. A large number of nights were allocated to this programme, but unfortunately not all of the nights were useful because of weather or technical problems. The dates with usable data are also listed in Table 1.

Because TNOs and Centaurs have typical drift rates ranging from one to a few arcsecs per hour, we used an exposure time that was short enough not to get elongated images of either the object or the field stars (depending on whether the telescope is tracked at sidereal or nonsidereal rate, respectively), but long enough to get a reasonable signal-to-noise ratio for the object. An exposure time of $300 \mathrm{~s}$ was short enough to avoid noticeable trailing under the best foreseeable seeing conditions, but long enough so that the sky background was the dominating noise source. In order to get an adequate signal-to-noise ratio, most of the observations were obtained with no filter. Therefore, unless otherwise noted, the observations consisted of sequences of $300 \mathrm{~s}$ integrations with no filter. The number of images obtained was therefore very high (except for two objects), on the order of a thousand. The typical seeing during the observations ranged from 1.2 arcsec to 2.8 arcsec, with the median around 1.5 arcsec. A fast readout $1024 \times 1024$ CCD was used in the runs prior to April 2003. The camera features are described in Ortiz et al. (2002), but we will briefly mention here that the field of view of the instrument was $7 \times 7$ arcmin and the pixel scale $0.41 \mathrm{arcsec} / \mathrm{pixel}$. The observations of the other runs were carried out by means of a larger format CCD. The CCD chip format for this camera is $2048 \times 2048$ pixels, and the total field of view is $7.8 \mathrm{arcmin} \times 7.8 \mathrm{arcmin}$. However, binning $2 \times 2$ was always used, giving a 0.46 arcsec/pixel scale. This scale was enough to have good point-spread function sampling even for the best seeing cases.
The data reduction process was identical for the two cameras. The images were bias-subtracted in the standard way and flat-fielded using a daily master flatfield frame, obtained as the median of a large set of dithered twilight images of the sky at blank fields. No cosmic ray removal algorithms were used, and we rejected the images in which a cosmic ray hit was close to the objects. Relative photometry using seven field stars was carried out by means of Daophot routines. The synthetic aperture used was typically 8 to 12 pixels in diameter, the smallest possible in order to get the highest signal to noise. Care was taken not to introduce spurious signals of faint background stars or galaxies in the aperture. In cases where the TNOs were close to faint stars or galaxies, the data were rejected.

Since the TNOs move relatively slowly, we were able to use the same field stars within each observing run. The typical error bars of the individual $300 \mathrm{~s}$ integrations are $\sim 0.1 \mathrm{mag}$ for the faintest targets we observed and around $\sim 0.06$ mag for the brightest ones. Both figures were considerably improved by averaging or "median averaging" the large amount of relative photometry data points. The approach of averaging is similar to using longer integrations times, but has the advantage that no trails are present in the images, comic ray hits are fewer, and images are also less smeared.

Due to the fact that the observations were carried out in a non-standard photometric band, the absolute calibration of the data would have a large uncertainty that could reach up to $0.5 \mathrm{mag}$ in some cases. Since the absolute calibration is irrelevant for this investigation where short-term variability of the object is obtained by using the stars in the field of view, no attempts were made to absolutely calibrate the data.

The time-resolved observations were inspected for periodicities by means of the Lomb technique (Lomb 1976) as implemented in Press et al. (1992), although we verified the results by using several other time-series analysis techniques (such as PDM and CLEAN) as implemented in the Starlink project. The reference stars were also inspected for short-term variability but none was found. We can thus be confident that no error was introduced by this.

Most of the objects were observed in more than one campaign, which allowed us to refine rotation periods, but in these 
Table 2. Summary of the photometric results. In the fourth column, we show the periodicities that correspond to the highest spectral power in the periodograms. Several values are given if different time-series analysis techniques favour different values. See the individual description for each object for a detailed description.

\begin{tabular}{|c|c|c|c|c|c|}
\hline & Object & $\begin{array}{c}\text { Amplitude } \\
\text { (mag) }\end{array}$ & $\begin{array}{l}\text { Photometric } \\
\text { period }^{a}(\mathrm{~h})\end{array}$ & Comments & $\begin{array}{c}\text { Reliability } \\
\text { code }^{b}\end{array}$ \\
\hline \multicolumn{6}{|l|}{ TNOs } \\
\hline & 2004 DW & $0.04 \pm 0.02$ & $10.08 \pm 0.01$ & Other possibilities exist & 2 \\
\hline & $2002 \mathrm{AW}_{197}$ & $0.08 \pm 0.07$ & $8.86 \pm 0.01$ & Other possibilities exist & 2 \\
\hline & $2003 \mathrm{AZ}_{84}$ & $0.10 \pm 0.04$ & $5.28 \pm 0.01$ or $6.76 \pm 0.01$ & $6.72 \mathrm{~h}$ according to & 2 \\
\hline & & & & Sheppard \& Jewitt (2003) & \\
\hline & $2003 \mathrm{VS}_{2}$ & $0.23 \pm 0.07$ & $3.71 \pm 0.01$ or $4.39 \pm 0.01$ & Likely spin period 8.77 & 2 \\
\hline & $2002 \mathrm{VE}_{95}$ & $0.08 \pm 0.04$ & $6.76 \pm 0.01,9.47 \pm 0.01$, or $7.36 \pm 0.01$ & & 2 \\
\hline & $2001 \mathrm{YH}_{140}$ & $0.19 \pm 0.04$ & $8.45 \pm 0.05$ & Other possibilities exist & 2 \\
\hline & $1996 \mathrm{TL}_{66}$ & $<0.12$ & $12.1 \pm 0.1$ & Non-confident detection & 1 \\
\hline \multicolumn{6}{|l|}{ Centaurs } \\
\hline & $2003 \mathrm{CO}_{1}$ & $0.10 \pm 0.05$ & $4.99 \pm 0.01$ & Other possibilities exist & 2 \\
\hline
\end{tabular}

${ }^{a}$ In this column we report the photometric period, understood as the most confident periodicity detected in the periodograms. This is the interval between two consecutive maxima. This would correspond to the actual spin period if the lightcurve is assumed to be single-peaked, which is usually the case for spherical bodies. However, elongated bodies would produce lightcurves with two maxima and two minima, and in those cases the actual spin period would be twice the photometric period reported in the table. In the text corresponding to each object of our sample, we discuss the possibility that each lightcurve is double-peaked and, therefore, that the table. ${ }^{b}$ Reliability code has been assigned according to the definition given in e.g. Lagerkvist et al. (1989). These authors defined a reliability scale for spin period determinations from 1 (tentative result) to 4 (multiple apparition coverage, pole position reported). A 2 stands for a reasonably secure result, based on over half coverage of the lightcurve.

cases the reference stars were different in the separated runs. This required especial treatment. In order to apply time-series analysis to different data sets of the same KBO, the usual approach is to absolutely calibrate each run using Landolt stars. Because an absolute calibration would not be very accurate introducing shifts of more than $\sim 0.04$ mag and, even if it were, the phase effect would introduce shifts to the data, so we preferred to use a different approach. We calculated the mean magnitude value in each separate run and found a multiplying factor for each campaign so that each run gave the same constant average value. This allowed us to level off any possible long-term or calibration shifts that introduce spurious signals into the periodograms. Once this was done, we carried out a regular periodogram analysis for the combined data set.

Typical $1 \sigma$ errors of the period determination in typical observing windows are on the order of $0.05 \mathrm{~h}$, neglecting the interfering effects of the aliases. In the cases in which we were able to use a longer time interval, the period determination is more precise; but as discussed in the following sections, the interfering effects of the aliases can produce ambiguities in the choice of the best period, and these uncertainties are indeed higher than a few hundredths of hour.

\section{Results}

The main photometric results are listed by object and summarised in Table 2.

\subsection{Reliable detections of periodic signals}

(90482) Orcus (formerly $2004 \mathrm{DW}$ ). The Lomb periodogram for this object (Fig. 1) shows several peaks. The peak with the highest spectral power (confidence level well above the 99.9\%)

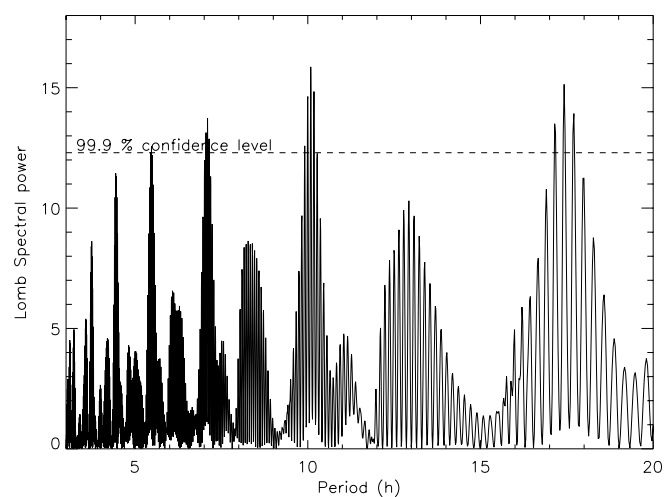

Fig. 1. Lomb periodogram for Orcus.

corresponds to a periodicity of $10.08 \mathrm{~h}$ ( 2.38 cycles/day). The periodogram shows another peak that exceeds the $99.9 \%$ confidence level, located at $17.43 \mathrm{~h}$ (1.38 cycles/day), probably an alias of the first periodicity; note the integer difference in this frequency with respect to the main peak frequency, expressed in cycles per day, as expected from an alias. In addition, the periodogram shows another high peak located at $7.09 \mathrm{~h}$ (3.38 cycles/day), exceeding the $99.9 \%$ confidence level as well and very likely another alias of the highest peak. Studying the photometric data with different time-series techniques (PDM, CLEAN, chi-square minimization, etc.), most of them show the highest spectral power at $10.08 \mathrm{~h}$. Only chi-square minimisation, by using harmonics larger than 2, gives the smallest chi-square for a periodicity of about $17.4 \mathrm{~h}$. Although most of the time-series analysis techniques tend to favor the photometric periodicity of $10.08 \mathrm{~h}$, it might happen that the correct period is $17.4 \mathrm{~h}$ and that $10.08 \mathrm{~h}$ is the alias. 

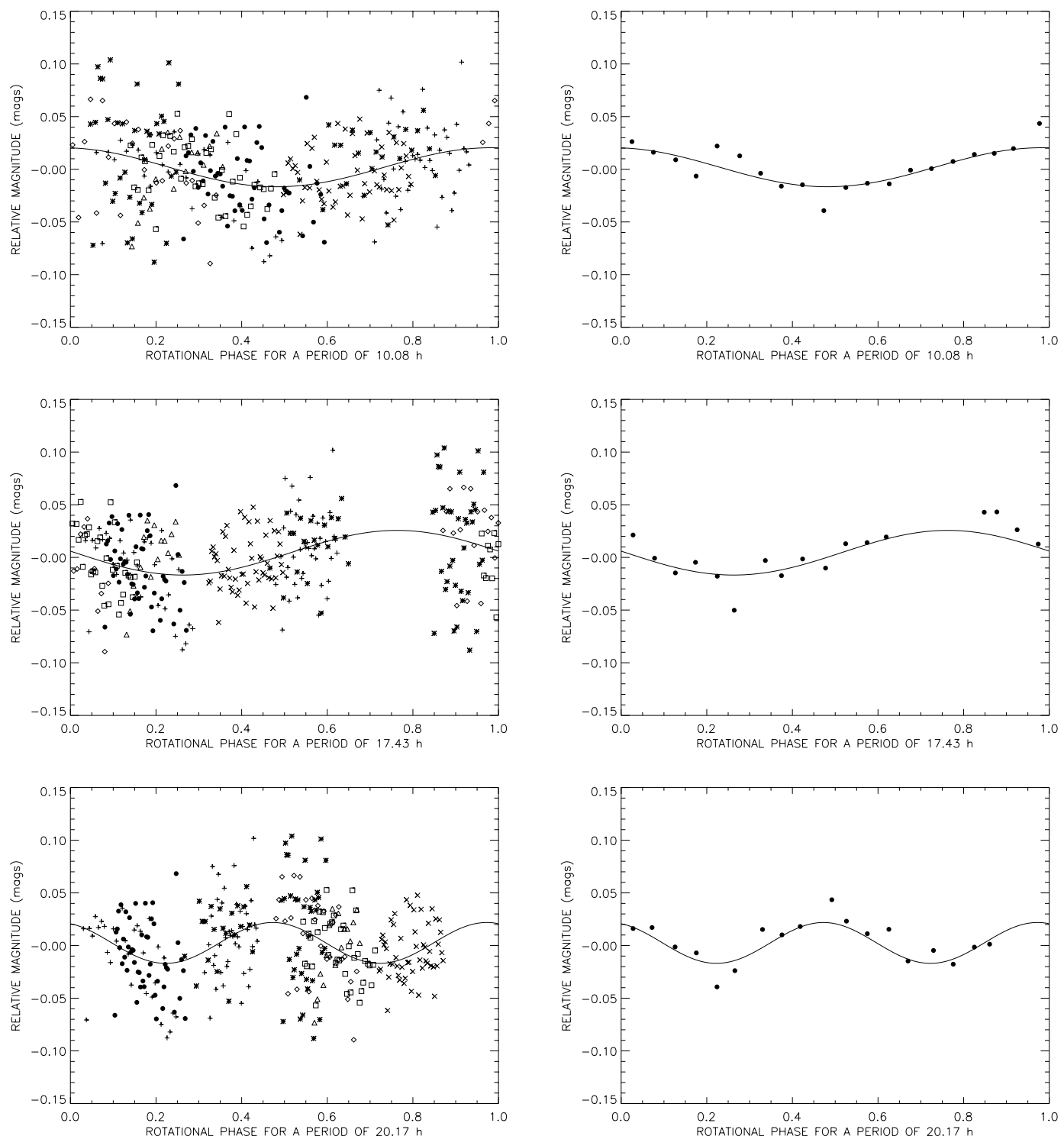

Fig. 2. Rotational phase curves for Orcus obtained by using different spin periods. The thick lines are simple sinusoidal fits of the photometric data. In the plots on the left, different symbols correspond to different dates. The plots on the right show the real tendency of the rotational phase curve. They were obtained by median averaging the actual photometric data in rotational phase bins of 0.05 .

Visual inspection of the rotational phase curves for the three above-mentioned periodicities and their doubles seems to favour $10.08 \mathrm{~h}$, but $17.43 \mathrm{~h}$ cannot be rejected. This can be seen in Fig. 2. According to the rotational phase curves, the spin period of this TNO could be $10.08 \mathrm{~h}$ or $17.43 \mathrm{~h}$, if the lightcurve is assumed to be single-peaked, or $20.16 \mathrm{~h}$ (twice the first periodicity), if the lightcurve is double-peaked. There are not enough data to try a spin period of $34.88 \mathrm{~h}$, twice $17.43 \mathrm{~h}$ because there is not enough rotation phase coverage. For all these periodicities, the amplitude of the lightcurve is $0.04 \pm 0.02 \mathrm{mag}$, obtained by fitting a simple sinusoidal curve to the photometric data. These results for amplitude and rotation period are consistent with Tegler et al. (2005) who state that 2004DW did vary on the order of $0.02 \mathrm{mag}$ in a $\sim 7 \mathrm{~h}$ interval.

$2002 A W_{197}$. The Lomb periodogram of this object shows several peaks with a confidence level higher than $99.99 \%$. The highest peak (see Fig. 3 ) is located at $8.86 \mathrm{~h}$ ( 2.71 cycles/day).

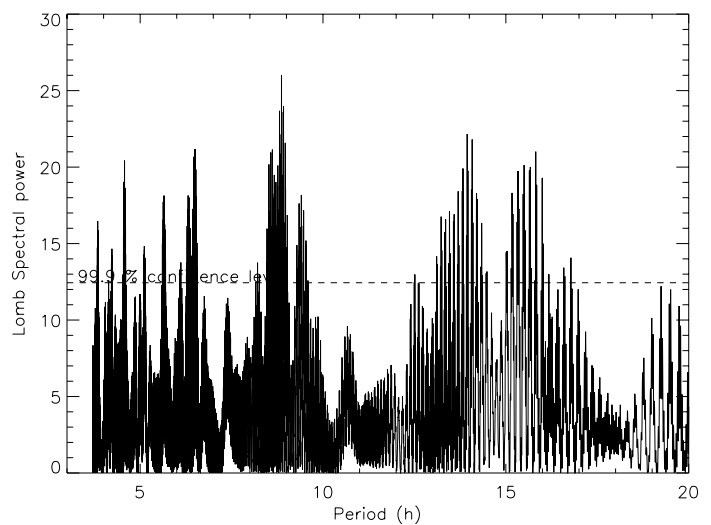

Fig. 3. Lomb periodogram for $2002 \mathrm{AW}_{197}$.

This peak is accompanied by two likely aliases located at $13.94 \mathrm{~h}$ ( 1.72 cycles/day) and at $6.49 \mathrm{~h}$ (3.69 cycles/day), both 

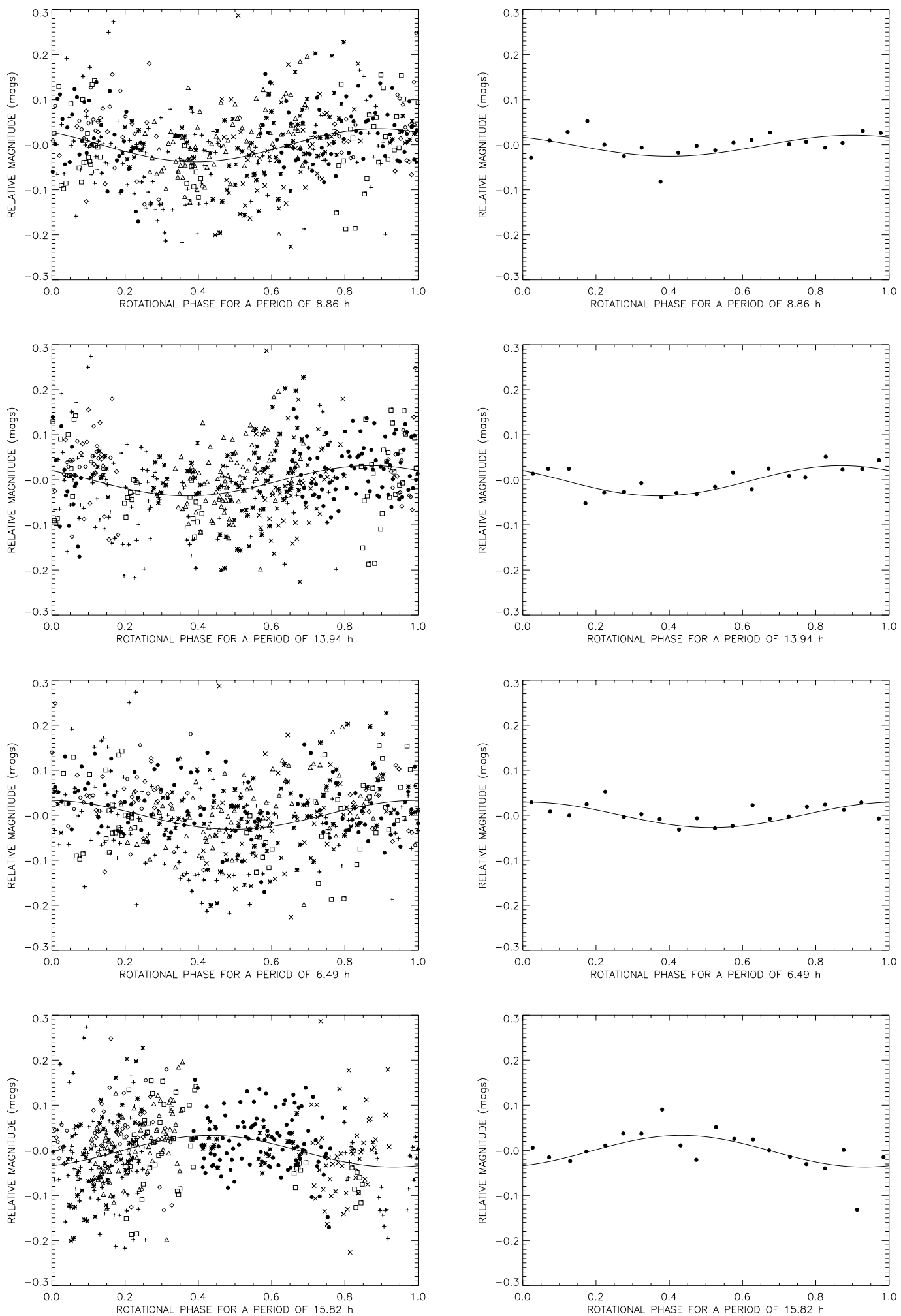

Fig. 4. Rotational phase curves for $2002 \mathrm{AW}_{197}$ obtained by using different spin periods. The thick lines are simple sinusoidal fits of the photometric data. In the plots on the left, different symbols correspond to different dates. The plots on the right show the real tendency of the rotational phase curve. They were obtained by median averaging the actual photometric data in rotational phase bins of 0.05 .

with a smaller spectral power than for the first periodicity. The periodogram also shows a highly confident peak at $15.82 \mathrm{~h}$ (1.52 cycles/day). The light curves phased to the different possible rotation periods are shown in Fig. 4. The plots do not clearly favour any of the $8.86 \mathrm{~h}$ or $13.94 \mathrm{~h}$ possibilities. The other time-series analysis techniques also favor the periodicity 


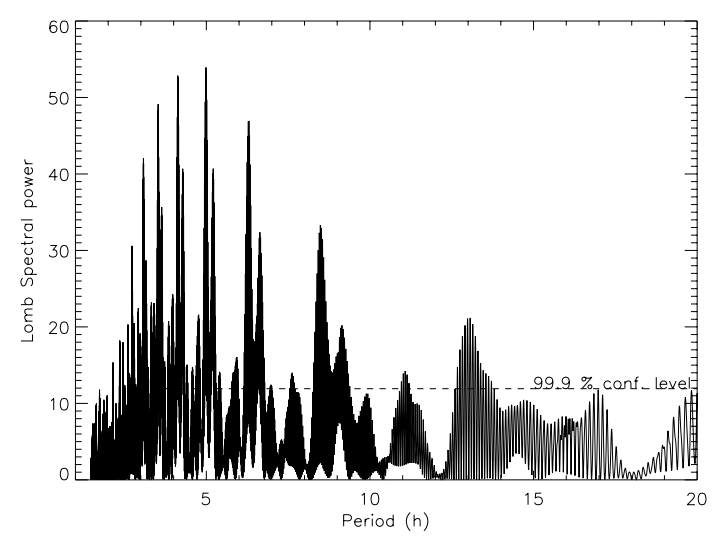

Fig. 5. Lomb periodogram for $2003 \mathrm{CO}_{1}$.

of $8.87 \mathrm{~h}$, for which the highest/deepest spectral power is always obtained. Thus, it can be said that periodograms tend to favor a spin period of $8.86 \mathrm{~h}$, if the lightcurve is assumed to be single-peaked, or $17.74 \mathrm{~h}$ if it is double-peaked. The amplitude of the lightcurves, determined by fitting a simple sinusoidal curve, is $0.08 \pm 0.03 \mathrm{mag}$.

$2003 \mathrm{CO}_{1}$. The time-series analysis of the photometric data for this object shows a very confident peak (significance higher than $99.99 \%)$ located at $4.99 \mathrm{~h}$ (4.81 cycles/day). The periodogram (Fig. 5) also shows some peaks (likely aliases of the highest peak, with a spectral power slightly lower than that of the main periodicity) located at $4.13 \mathrm{~h}(5.81 \mathrm{cycles} /$ day $), 3.53 \mathrm{~h}$ (6.81 cycles/day), and $6.30 \mathrm{~h}$ (3.81 cycles/day). The PDM and CLEAN methods also favour the $4.99 \mathrm{~h}$ peak. Visual inspection of the rotational phase curve (Fig. 6) confirms that this periodicity is likely, but we cannot conclude if the lightcurve is single or double-peaked. Rotational phase curves also show a good fit for a period of $6.30 \mathrm{~h}$ and $4.13 \mathrm{~h}$, while $3.53 \mathrm{~h}$ seems to be discarded. The amplitude of the lightcurve ranges from $0.10 \pm 0.05$ for a period of $4.99 \mathrm{~h}$ to $0.13 \pm 0.04$ for the period of $6.30 \mathrm{~h}$.

$2001 \mathrm{YH}_{140}$. Analysis of the data (Fig. 7) for this TNO shows a confident peak $(99.4 \%)$ located at $8.45 \mathrm{~h}$ ( 2.84 cycles/day). This peak is flanked by possible aliases (with a spectral power and significance slightly lower than that of the highest peak) located at $12.99 \mathrm{~h}$ ( 1.84 cycles/day) and $6.22 \mathrm{~h}$ ( 3.85 cycles/day). Visual inspection of the rotation phase curves for the different periods does not clearly favor any of the possibilities (Fig. 8). By fitting a sinusoidal curve to the photometric data, we obtained an amplitude of the lighturve of $0.19 \pm 0.04$ for all three periodicities.

\subsection{Conflicting results and non-detected periodicities}

$2003 A Z_{84}$. Sheppard \& Jewitt (2003) (henceforth S\&J) have already obtained photometric data showing a very confident periodicity of $6.72 \pm 0.05 \mathrm{~h}$ (3.55 cycles/day). Their rotational phase curve for this period was excellent (Fig. 2 in Sheppard \& Jewitt 2003) with a peak-to-peak amplitude of $0.14 \pm 0.03$ mag.

Our complete data set for this object consists of two observational sets separated by almost one year. The first run covers the end of Dec. 2003 up to Jan. 2004. The second data set was taken in Dec. 2004. The Lomb periodogram of the complete data (noisier than those of S\&J, but cover a much larger time span), shows several peaks with a very high confidence level (Fig. 9). The highest peak, with a confidence level exceeding the $99.9 \%$, is located at $5.28 \mathrm{~h}$ (4.55 cycles/day). This peak is flanked by two likely aliases with very similar spectral power located at $4.32 \mathrm{~h}(5.55$ cycles/day) and $6.76 \mathrm{~h}$ (3.55 cycles/day), the latter periodicity being nearly coincident with the photometric periodicity reported by S\&J. We have also separately analysed the two data sets forming our complete data set. Concerning the first data set, the Lomb spectrogram gives the same results (with different spectral power, but all the peaks holding the significance level) as the complete data set. As for the second data set alone (which contains data with less scatter and covering a larger time span than the first run), the Lomb periodogram (Fig. 9) favours the peak located at $6.76 \mathrm{~h}$ (3.55 cycles/day), for which the maximum spectral power is obtained. Analysis of the various sets with the PDM technique gives similar results to those obtained with the Lomb technique.

We built the rotational phase curves for all the aforementioned periodicities, along with that of S\&J and their doubles for the three data sets. Visual inspection of the lightcurves allows us to discard most of them. The complete data set best-phase curve is for a period of $10.56 \mathrm{~h}$, twice the periodicity with the highest spectral power found in the spectrogram (Fig. 10). The amplitude is $0.08 \pm 0.04 \mathrm{mag}$. For the complete set, a period of $6.72 \mathrm{~h}$ or $6.76 \mathrm{~h}$ or their doubles yield unacceptable rotational phase curves. Nevertheless, the individual data sets give acceptable rotational phase curves for a period of $6.76 \mathrm{~h}$, the second data set slightly better than the first (Fig. 10). For these latter cases, the amplitude is $0.10 \pm 0.04 \mathrm{mag}$, which is also compatible with the amplitude measured by $\mathrm{S} \& \mathrm{~J}$.

In summary, the periodogram and rotational phase curve of the best data set suggest a period of $6.76 \mathrm{~h}$, which is very close to S\&J; but if the other data set is included, the preferred period would be $10.56 \mathrm{~h}$. It may well be that a problem with the first data set is forcing the anomalous result in comparison with S\&J.

$2003 V_{2}$. The Lomb periodogram for this object (Fig. 11) shows a very confident peak at $3.71 \mathrm{~h}$. (6.47 cycles/day). The periodogram also shows another confident peak at $4.39 \mathrm{~h}$. (5.47 cycles/day), very likely an alias of the highest peak. Other significant peaks are identified as harmonics of the aforementioned periodicities. The Clean technique and chi-square minimisation by using single harmonic fitting also show a strong peak at $3.71 \mathrm{~h}$. Nevertheless, the PDM technique and chi-square minimisation by using harmonics larger than 1 favor the peak located at $4.39 \mathrm{~h}$. Visual inspection of the rotational phase curves (shown in Fig. 12) for both periods (including their doubles) seems to indicate that the lightcurve is doublepeaked with a period of $8.77 \mathrm{~h}$. (twice $4.39 \mathrm{~h}$.), as photometric data for different dates seem to fit slightly better than for the other periodicities. Nevertheless, we cannot definitely favour or discard any of the these periodicities. For all of these periodicities, the amplitude of the lightcurve is estimated by fitting a single sinusoidal curve as $0.23 \pm 0.07 \mathrm{mag}$. 

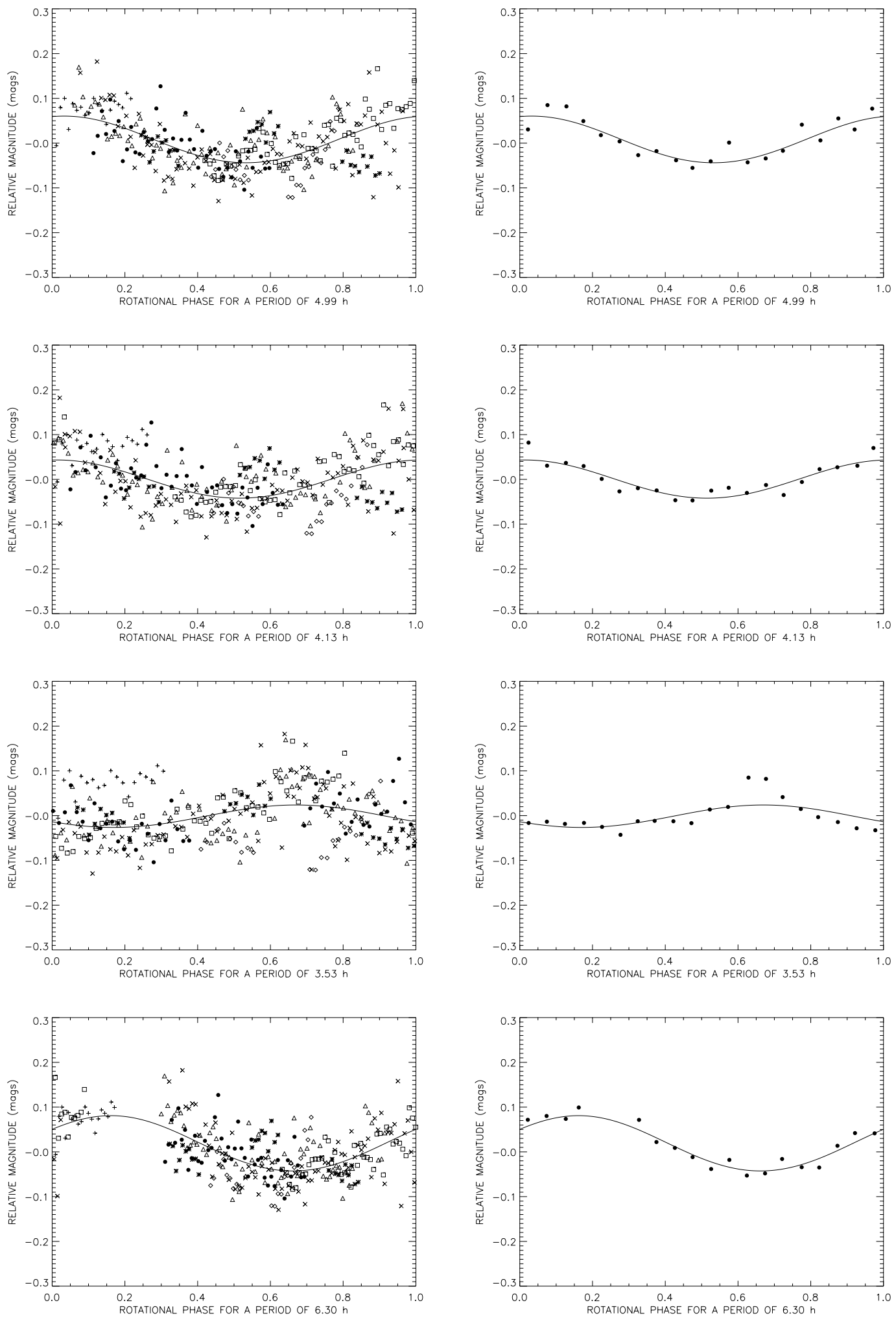

Fig. 6. Rotational phase curves for $2003 \mathrm{CO}_{1}$ obtained by using different spin periods. The thick lines are simple sinusoidal fits of the photometric data. In the plots on the left, different symbols correspond to different dates. The plots on the right show the real tendency of the rotational phase curve. They were obtained by median averaging the actual photometric data in rotational phase bins of 0.05 .

$2002 V E_{95}$. For this object, the different time series analysis techniques give different results. The Lomb periodogram shows two strong peaks, with very similar spectral power, located at $6.76 \mathrm{~h}$ ( 3.55 cycles/day) and $9.47 \mathrm{~h}(2.53$ cycles/day $)$. Presumably, one peak is an alias of the other one. Both peaks have a significance level that is higher than $99.9 \%$. As can be 


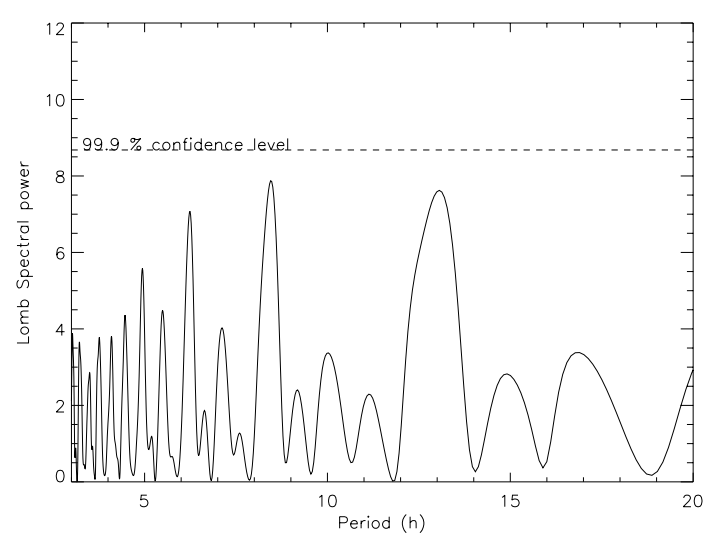

Fig. 7. Lomb periodogram for $2001 \mathrm{YH}_{140}$.

seen in Fig. 13, apart from these two peaks, there are many more peaks above the $99.9 \%$ significance level, and all of them should correspond to photometric periodicities. The PDM technique gives a strong local minimum at $6.88 \mathrm{~h}$ (near one of the highest periodicities detected with the Lomb technique), but it shows the deepest peak for a periodicity of $7.36 \mathrm{~h}$. Finally, the Clean technique gives the maximum spectral power for a periodicity of $6.88 \mathrm{~h}$. The available photometric data are noisy and rotational phase curves (even median averaging) do not allow us (see Fig. 14) to favor or discard any of the above-mentioned periodicities. Therefore, our results concerning the photometric periodicity for this object are uncertain. In all cases, the amplitude of the variability is below $0.08 \pm 0.04 \mathrm{mag}$, consistent with Sheppard \& Jewitt (2003), who found no variability at the 0.06 mag. level on this object.

$1996 T_{66}$. For $1996 \mathrm{TL}_{66}$, the photometric analysis of the data gave no reliable detection of periodicities. For this object, the periodogram shows a peak located at $12.1 \mathrm{~h}$ with a confidence level of $64.2 \%$. The periodogram (See Fig. 15) also shows peaks at $8 \mathrm{~h}$ and $6 \mathrm{~h}$, both probable aliases of the highest peak. Rotational phase curves for these periods (and their doubles) do not allow us to favour or discard any of them. In any case, the peak-to-peak variability must be smaller than 0.12 mag.

\section{Discussion}

Sheppard \& Jewitt (2002) presented a systematic investigation of rotational lightcurves of large TNOs using their own results and compiling results from other variability studies. From their study, they found that $32 \%$ of the sample displays lightcurve amplitudes larger than $0.15 \mathrm{mag}$ (which is a plausible limit for variability being produced by irregular shape) and that $23 \%$ of their sample shows amplitudes larger than $0.4 \mathrm{mag}$, denoting that there could be a significant fraction of highly irregular bodies among large TNOs. Even when including the results that meet the criteria of $\mathrm{H}$ being smaller than $7.5 \mathrm{mag}$ from Ortiz et al. (2003a,b, 2004), those of Sheppard \& Jewitt (2003), and those of this work, the statistics are hardly modified. Now, 10 out of 32 objects (31\%) show amplitudes larger than $0.15 \mathrm{mag}$, but only $16 \%$ display amplitudes larger than 0.4 mag. Sheppard \& Jewitt (2002) propose that large-amplitude, fast-rotating bodies could be rubble piles distorted by rotation. This idea is partially based on the fact that large bodies (larger than $100 \mathrm{~km}$ ) residing in the inner part of the Kuiper-Edgeworth belt have most likely been converted into rubble piles by collisions, according to Davis \& Farinella (1997).

All the bodies studied in this work (except the centaur) are larger than $100 \mathrm{~km}$ (assuming a plausible albedo of 0.09); therefore, they could be collision-induced strengthless rubble piles. Following the reasoning of Sheppard \& Jewitt (2002), we find the objects could be elongated bodies due to their comparatively fast rotation. Under this hypothesis, we have calculated the minimum axis ratio from their lightcurves, assuming variability is produced by elongation and calculated by assuming an aspect angle of $90^{\circ}$. In addition, as Sheppard \& Jewitt (2002) did for Varuna, we also calculated the density of the objects using the tables provided by Chandrasekhar (1987) and assumed they are strengthless bodies that adopt the shape of a Jacobi ellipsoid due to rotation. Moreover, we also calculated the minimum density to be stable under rotation, to avoid spontaneous break-up if the bodies are strengthless. Axis ratios and densities are shown in Table 3 in Cols. 3, 5, and 6. Most of the objects of this study show small amplitudes (only two exceed the above-mentioned limit of $0.15 \mathrm{mag}$ ), leading to relatively small axis ratios. If these bodies were indeed elongated, this could be the result of being observed nearly pole-on. Concerning density, it is mainly controlled by the spin period, barely depending on the axis ratio. The objects of this study spin slower than Varuna (for which a density of $1000 \mathrm{~kg} / \mathrm{m}^{3}$ was estimated by Sheppard \& Jewitt 2002), so they would have comparatively small densities. In this study, most of the bodies would have densities even smaller than $500 \mathrm{~kg} / \mathrm{m}^{3}$, a value estimated for Pholus by Tegler et al. (2005) by using the same approach. Only $2003 \mathrm{VS}_{2}$ could have a density between 500 and $1000 \mathrm{~kg} / \mathrm{m}^{3}$. On the other hand, it can be seen that all the bodies, even if they are strengthless rubble piles, would be stable under rotation for their plausible spin periods, as minimum densities required to avoid rotational break-up are very small (most require a minimum density smaller than $100 \mathrm{~kg} / \mathrm{m}^{3}$ ), much smaller than estimated cometary densities (e.g. Davidsson \& Gutiérrez 2005).

Indeed, these values for the densities should be taken as illustrative and useful just for comparison with previous estimates. Certainly, considering TNOs as fluid-like bodies is an oversimplification, as they must have some internal strength. Otherwise, the objects could be considered as compact solid bodies. It is interesting to note that these bodies are rotating relatively fast and that, under the assumption of being compact solid objects, the rotation would produce internal strengths that could yield internal fractures, although the "fragments" might be held together by gravity; i.e. the bodies are rotationally stable. By using the expressions given by Davidsson (2001), it is possible to estimate the material strength necessary to stay intact, withstanding tensile splitting and shear fracturing produced by rotation. In Col. 7 of Table 3, we show the minimum material strength needed to withstand shear fracture assuming the bodies have a density of $1000 \mathrm{~kg} / \mathrm{m}^{3}$. It can be seen that all the bodies of this study would need considerably large material strength, in order to withstand shear fracturing, ranging from 

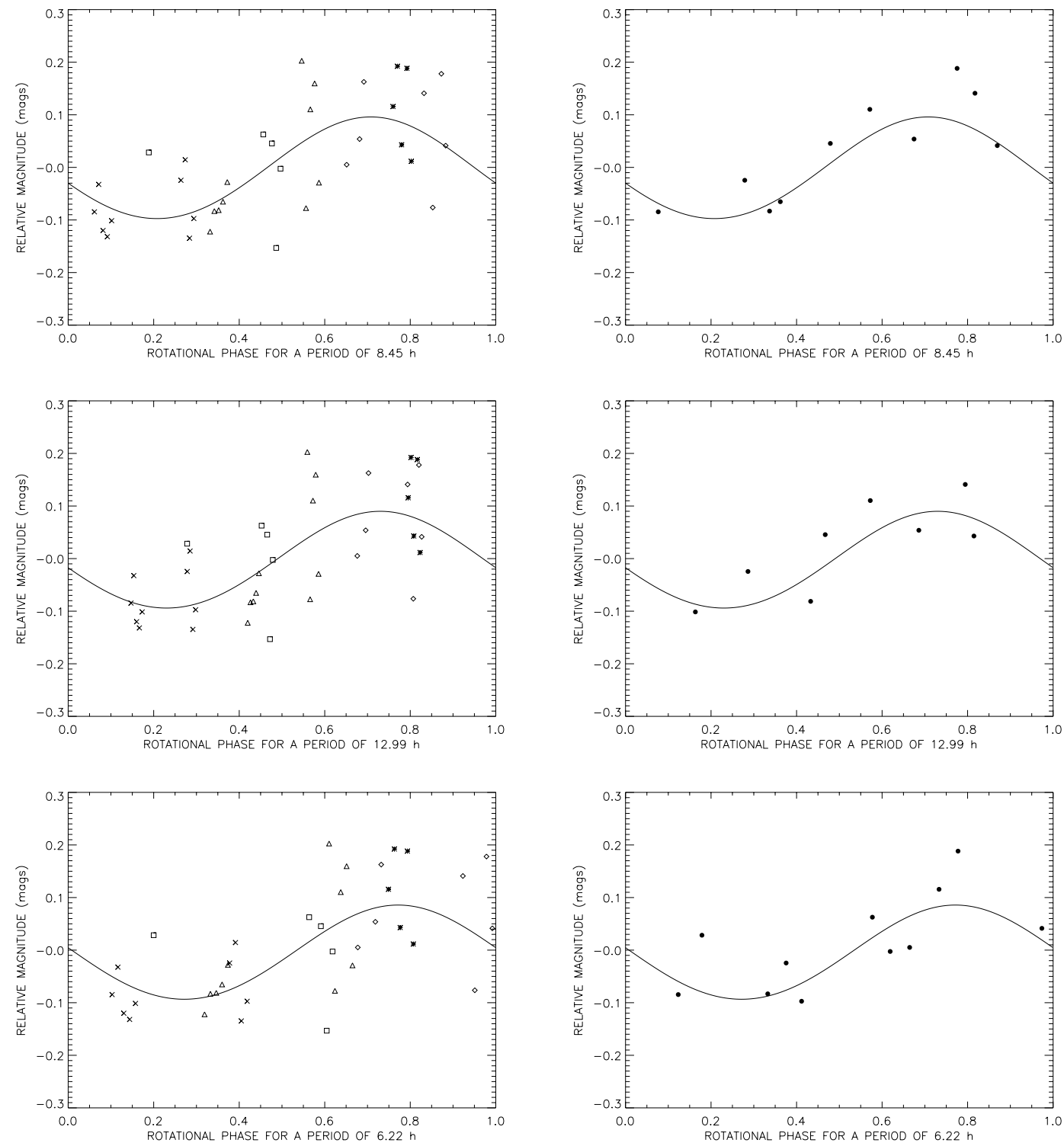

Fig. 8. Rotational phase curves for $2001 \mathrm{YH}_{140}$ obtained by using different spin periods. The thick lines are simple sinusoidal fits of the photometric data. In the plots on the left, different symbols correspond to different dates. The plots on the right show the real tendency of the rotational phase curve. They were obtained by median averaging the actual photometric data in rotational phase bins of 0.05 .
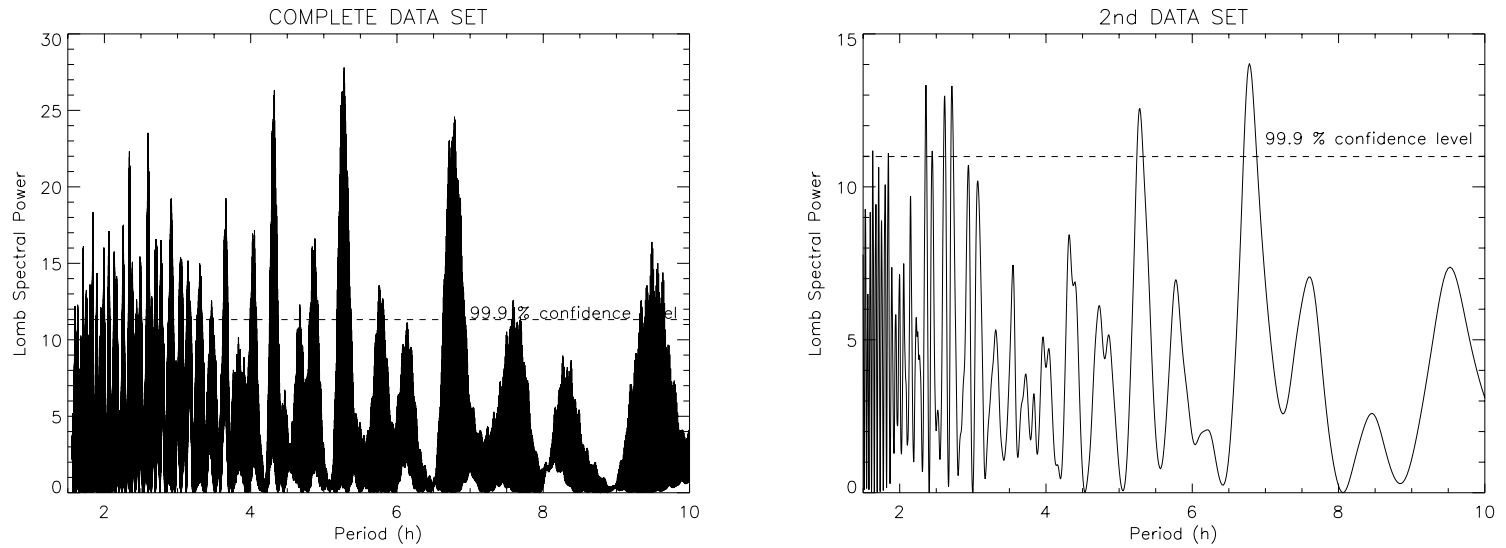

Fig. 9. Lomb periodograms for $2003 \mathrm{AZ}_{84}$. On the left, the periodogram of the complete data set is shown. On the right, we show the periodogram of the second data set. 

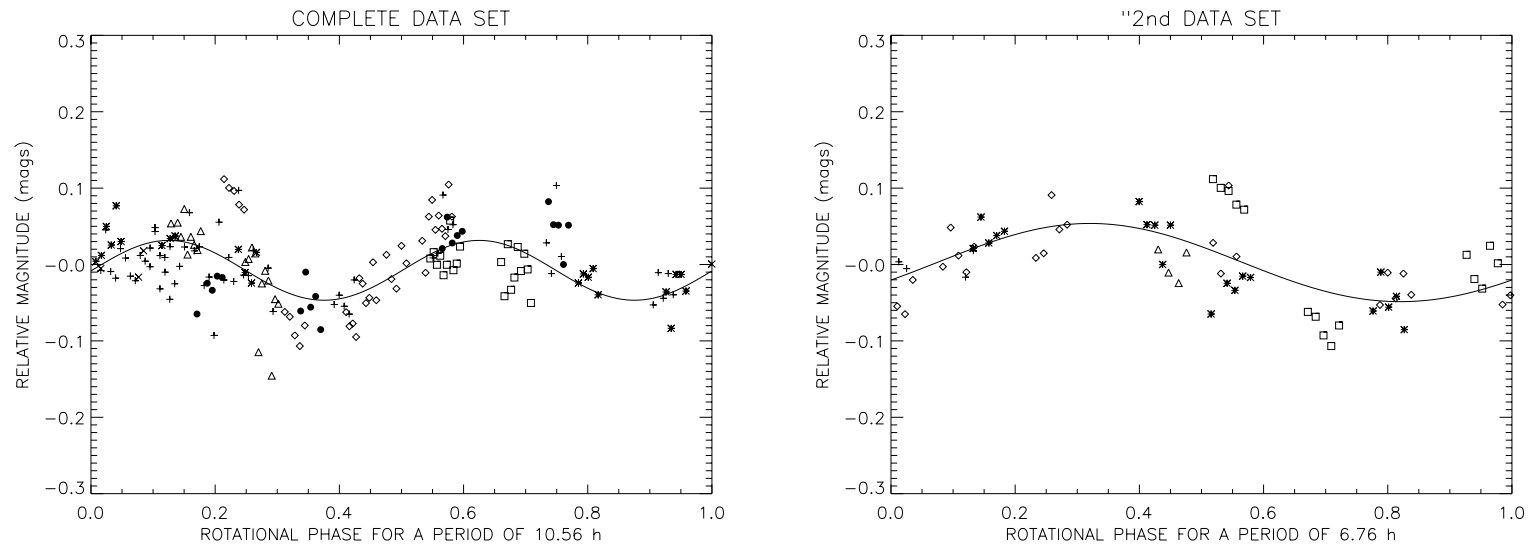

Fig. 10. Rotational phase curves of $2003 \mathrm{AZ}_{84}$ for periods of $10.56 \mathrm{~h}$ (left, for the complete data set) and $6.76 \mathrm{~h}$ (right, for the second data set).

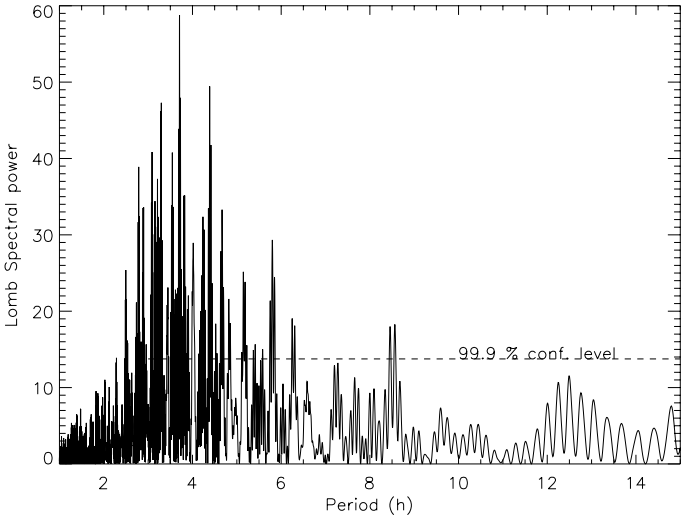

Fig. 11. Lomb periodogram for $2003 \mathrm{VS}_{2}$.

several $\mathrm{kPa}$ to several MPa. At present, little is known about the internal strengths of Solar System bodies, but, in any case, the values shown in Table 3 are several orders of magnitude higher than the expected values for internal strength of comets (supposed to be "products" of TNOs). If TNOs do not have as high material strength as in Table 3 then the bodies would be structurally damaged and may well become rubble piles due just to rotation, with less need for collisions or smaller impacts than usually thought. This possibility could have implications on collisional evolution models, size distribution of TNOs, and the inventory of binary objects. The fact that TNOs could be strengthless bodies held together by gravity is also consistent with recent observations regarding the size distribution of the smallest bodies detectable nowadays, as pointed out by Pan \& Sari (2005).

Due to rotation, large TNOs (either if they were preshattered or not) could easily be disrupted by small impactors. A similar reasoning for main belt asteroids has been followed by Housen (2004), who performed preliminary laboratory experiments and concluded that the spin plays an important role, as it significantly affects the conditions required for collisional disruption.

On the other hand, if the bodies spin up and fission (for some reason such as a tidal interaction in a close encounter with a large body), a binary can result. This has been proposed as a binary formation mechanism for NEAs (Margot et al. 2002) based on numerical (Bottke \& Melosh 1996; Richardson et al. 1998) and analytical models (e.g. Sridhar \& Tremain 1992). Encounters with nearly planetary-sized bodies are currently thought to be unlikely, but might have been frequent in the early ages of the trans-neptunian belt.

In addition, impacts with a small relative velocity could result in fragments coming apart from the parent body with small relative velocity (smaller than the scape velocity) and remaining as a satellite near the parent body. The formation of binaries from collisions has been investigated from the point of view of energy by Stern (2002), who concluded that $1 \%$ of binary TNOs could arise from collisions provided that the albedos of the bodies are larger than $\sim 0.15$, a value that should be sensitive to the fraction of impactor energy that is partitioned into kinetic energy of the ejecta, $f_{\mathrm{KE}}$, which Stern (2002) took as 0.2 and 0.05 for the two extreme cases. The fact that the objects are rotating and/or preshattered could ease some of the energetics needed in the simple treatment by Stern, so the impacting bodies could be smaller than proposed by Stern (2002) in order to produce sizable satellites. This would have two immediate implications: the percentage of binaries could be larger than $1 \%$ (which appears to be the case, e.g. Noll et al. 2002) and the albedos would not have to be as large. In addition, the use of densities close to the ones derived here (under the assumption of Jacobi ellipsoids), rather than the assumed $2000 \mathrm{~kg} / \mathrm{m}^{3}$ by Stern (2002), implies that smaller bodies can generate sizable satellites. Also, the fact that the bodies were pre-shattered could imply a larger ratio of impactor energy that is partitioned into kinetic energy of the ejecta than the maximum adopted by Stern (2002), because the $f_{\mathrm{KE}}$ references given by Stern apply only to impacts with monolithic targets. This would also ease the minimum impactor mass giving rise to sizable satellites. Certainly, these qualitative reasonings are speculative and quantitative investigations should be addressed before reaching conclusions on the definite role of rotation on collisions.

\section{Summary and conclusions}

In this paper we report confident short-term variability of seven TNOs and a centaur, presumably due to rotation. All of them show lightcurves with small amplitudes, smaller than 0.25 mag. 

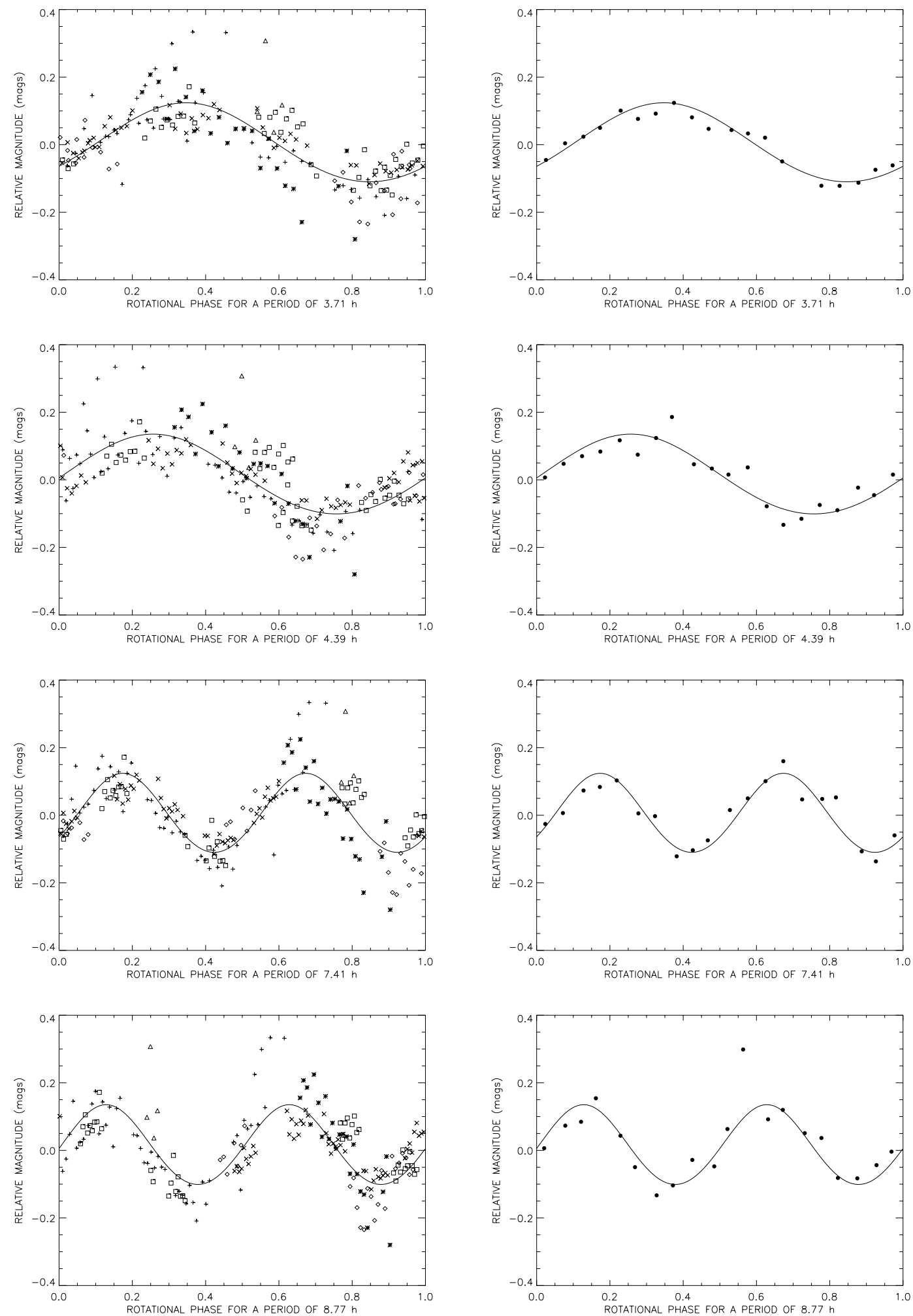

Fig. 12. Rotational phase curves for $2003 \mathrm{VS}_{2}$ obtained by using different spin periods. The thick lines are simple sinusoidal fits of the photometric data. In the plots on the left, different symbols correspond to different dates. The plots on the right show the real tendency of the rotational phase curve. They were obtained by median averaging the actual photometric data in rotational phase bins of 0.05 .

We were not able to determine if the variability is produced by albedo variegations or by elongated shape. Assuming that the bodies are strengthless rubble piles, all of them are presumably stable under rotation, as the minimum densities needed to avoid rotational break-up are very small, and most of them being smaller than $100 \mathrm{~kg} / \mathrm{m}^{3}$. Only $2003 \mathrm{VS}_{2}$, the fastest 
Table 3. Some of characteristics and properties of the bodies showing short-term variability, some of them deduced from their rotational states. The size, estimated from its absolute magnitude assuming an albedo of 0.09 , is shown in Col. 2. In Col. 3, we show the minimum axis ratio, estimated from the amplitude of the lightcurves assuming that the variability is produced by the elongation of the body viewed from an aspect angle of $90^{\circ}$. In Col. 4 , we show the different spin periods, according to the periodograms discussed in the previous section. Column 5 shows the densities of the objects, assuming they are strengthless, selfgravitating bodies distorted by rotation as Jacobi ellipsoids. These values have been estimated by using the tables provided by Chandrasekhar (1987). Densities are calculated only for periods twice the photometric periodicity suggested by the periodograms of the previous section. Column 6 displays the minimum density required to avoid spontaneous break-up, calculations performed by using the equations given in Davidsson (2001). Column 7 shows the minimum material strength necessary to withstand shear fracture and stay intact, assuming that the objects are compact bodies.

\begin{tabular}{|c|c|c|c|c|c|c|c|}
\hline \multirow[t]{2}{*}{ Object } & \multirow[t]{2}{*}{$\begin{array}{c}\text { Diameter } \\
(\mathrm{km})\end{array}$} & \multirow[t]{2}{*}{$\begin{array}{l}\text { Minimum } \\
\text { axis } \\
\text { ratio }\end{array}$} & \multirow[t]{2}{*}{$\begin{array}{l}\text { Spin } \\
\text { period } \\
(\mathrm{h})\end{array}$} & \multirow[t]{2}{*}{$\begin{array}{l}\text { Density }\left(\mathrm{kg} / \mathrm{m}^{3}\right) \\
\quad \text { (assuming } \\
\text { Jacobi ellipsoids) }\end{array}$} & \multicolumn{2}{|c|}{$\begin{array}{c}\text { Minimum } \\
\text { density } \\
\text { to avoid breakup }\left(\mathrm{kg} / \mathrm{m}^{3}\right)\end{array}$} & \multirow[t]{2}{*}{$\begin{array}{c}\text { Minimum } \\
\text { material strength }(\mathrm{kPa}) \\
\text { for } \rho=1000 \mathrm{~kg} / \mathrm{m}^{3}\end{array}$} \\
\hline & & & & & Prolate & Spherical & \\
\hline \multirow[t]{3}{*}{2004 DW } & 1600 & $1.04: 1$ & 10.08 & - & - & 100 & 2000 \\
\hline & & & 17.43 & - & - & 35 & 800 \\
\hline & & & 20.16 & 95 & 30 & 30 & 600 \\
\hline \multirow[t]{2}{*}{$2002 \mathrm{AW}_{197}$} & 970 & $1.08: 1$ & 8.86 & - & - & 140 & 1100 \\
\hline & & & 17.74 & 125 & 40 & 35 & 280 \\
\hline \multirow[t]{4}{*}{$2003 \mathrm{AZ}_{84}$} & 730 & $1.10: 1$ & 5.28 & - & - & 435 & 1820 \\
\hline & & & 6.76 & - & - & 240 & 1110 \\
\hline & & & 10.56 & 350 & 105 & 100 & 455 \\
\hline & & & 13.52 & 215 & 65 & 60 & 280 \\
\hline \multirow[t]{4}{*}{$2003 \mathrm{VS}_{2}$} & 640 & $1.24: 1$ & 3.71 & - & - & 790 & 2800 \\
\hline & & & 4.39 & - & - & 560 & 2000 \\
\hline & & & 7.42 & 720 & 240 & 200 & 700 \\
\hline & & & 8.77 & 515 & 170 & 140 & 500 \\
\hline \multirow[t]{4}{*}{$2002 \mathrm{VE}_{95}$} & 380 & $1.08: 1$ & 6.77 & - & - & 240 & 300 \\
\hline & & & 9.47 & - & - & 120 & 150 \\
\hline & & & 13.54 & 212 & 65 & 60 & 75 \\
\hline & & & 18.94 & 108 & 30 & 30 & 40 \\
\hline \multirow[t]{3}{*}{$2001 \mathrm{YH}_{140}$} & 350 & $1.20: 1$ & 8.45 & - & - & 150 & 163 \\
\hline & & & 12.99 & - & - & 65 & 69 \\
\hline & & & 16.9 & 140 & 45 & 40 & 40 \\
\hline \multirow[t]{4}{*}{$2003 \mathrm{CO}_{1}$} & 75 & $1.10: 1$ & 4.99 & - & - & 440 & 21 \\
\hline & & $1.13: 1$ & 6.30 & - & - & 275 & 13 \\
\hline & & $1.10: 1$ & 9.98 & 400 & 120 & 100 & 5 \\
\hline & & $1.13: 1$ & 12.60 & 250 & 75 & 70 & 3 \\
\hline
\end{tabular}

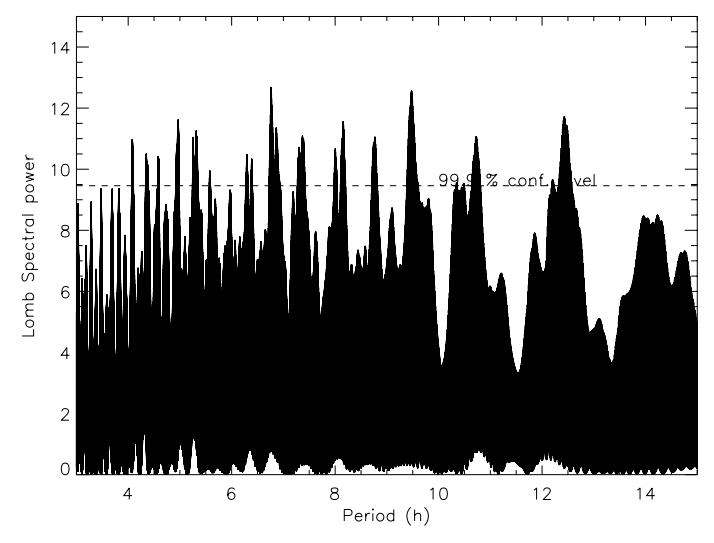

Fig. 13. Lomb periodogram for $2002 \mathrm{VE}_{95}$. rotator of this sample, would require a comparatively high density to be stable under rotation. Assuming that the bodies are irregularly shaped rubble piles and considering them as a fluidlike material, their spin periods and amplitudes suggest that their densities would range between 100 and $700 \mathrm{~kg} / \mathrm{m}^{3}$. On the other hand, if it is assumed that they are compact bodies with internal cohesion, it is found that substantial internal strength is needed in order to withstand shear fracture due to rotation. This points out that large TNOs rotating with similar spin periods to the ones detected might be structurally damaged, or even pre-shattered. In particular, this could have a strong impact on collisional evolution models, current size distributions, and binary formation. 

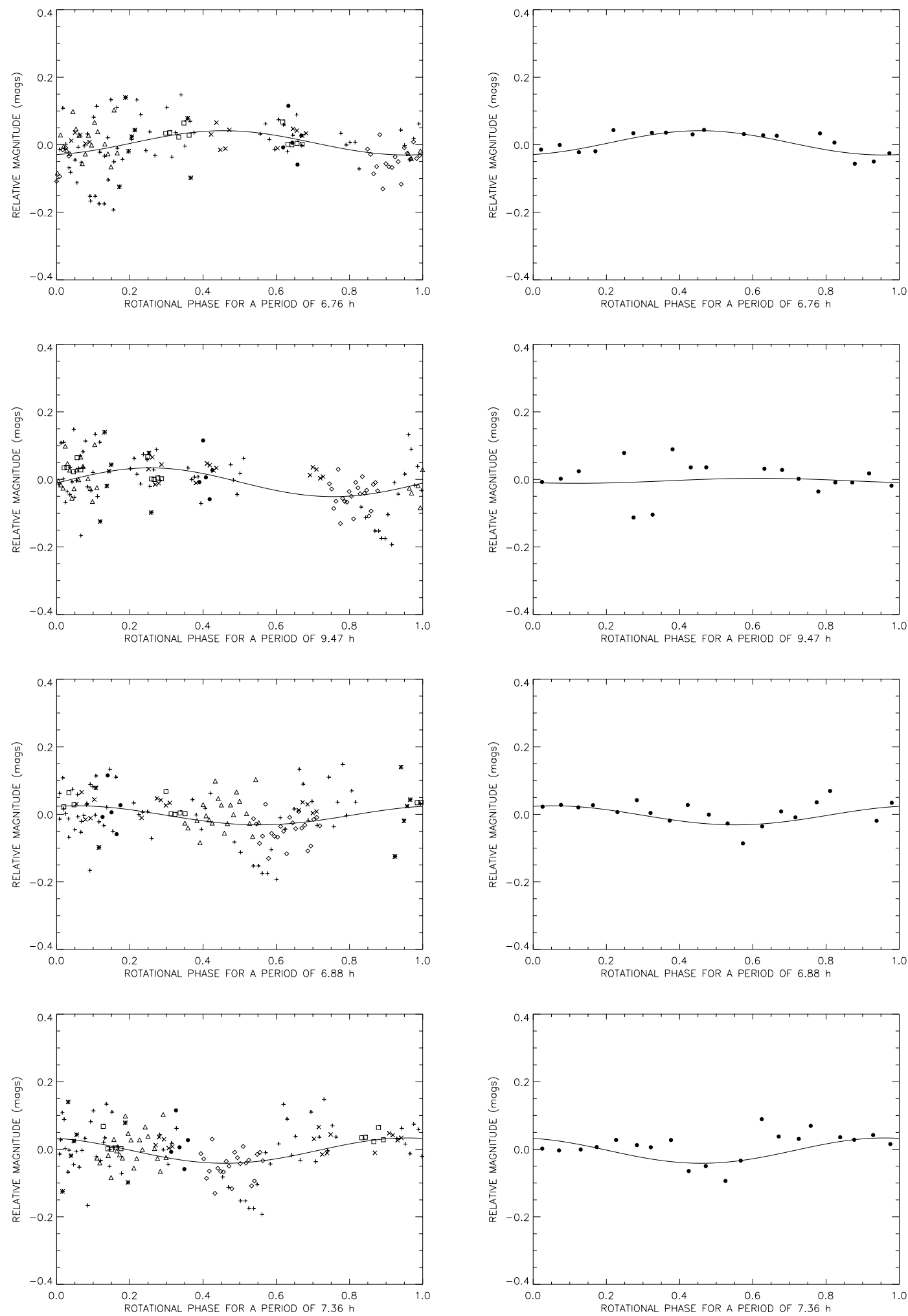

Fig. 14. Rotational phase curves for $2002 \mathrm{VE}_{95}$ obtained by using different spin periods. The thick lines are simple sinusoidal fits of the photometric data. In the plots on the left, different symbols correspond to different dates. The plots on the right show the real tendency of the rotational phase curve. They were obtained by median averaging the actual photometric data in rotational phase bins of 0.05 .

Acknowledgements. We are grateful to the Sierra Nevada Observatory staff. This research was based on data obtained at the Observatorio de Sierra Nevada, which is operated by the Instituto de Astrofísica de Andalucía, CSIC. This work was supported by contracts
AYA-2002-00382 and AYA-2004-03250. European FEDER funds for these contracts are also acknowledged. We also thank an anonymous referee for his helpful comments. 


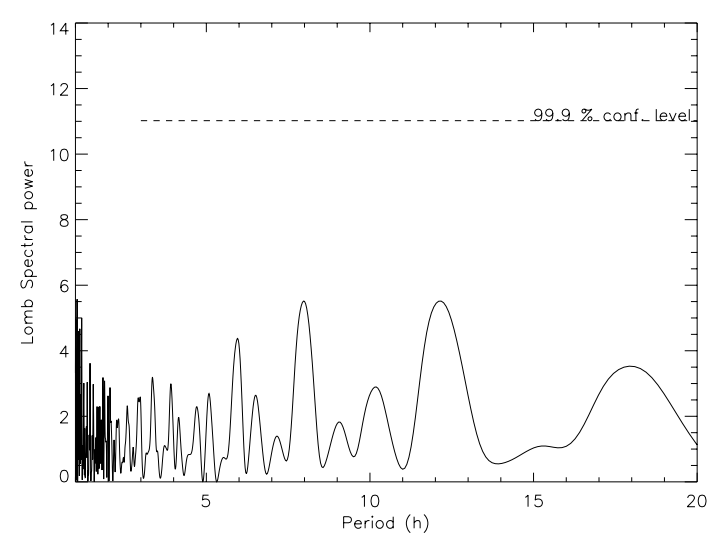

Fig. 15. Lomb periodogram for $1996 \mathrm{TL}_{66}$.

\section{References}

Barucci, M. A., Boehnhardt, H., Dotto, E., et al. 2002, A\&A, 392, 335

Bauer, J. M., Meech, K. J., Fernndez, Y. R., Farnham, T. L., \& Roush, T. L. 2002, PASP, 114, 1309

Belskaya, I. N., Barucci, A. M., \& Shkuratov, Y. G. 2003. Earth, Moon and Planets, 92, 201

Boehnhardt, H., Delsanti, A., Barucci, A., et al. 2002, A\&A, 395, 297

Bottke, W. F., \& Melosh, H. J. 1996. Icarus, 124, 372

Chandrasekhar, S. 1987, Ellipsoidal Figures of Equilibrium (New York: Dover Publications, Inc.)

Delsanti, A., Peixinho, N., Doressoundiram, A., et al. 2004, BAAS, 36, \#17.03

Doressoundiram, A., Peixinho, N., \& de Bergh, C. 2002, AJ, 124, 2279

Doressoundiram, A., Peixinho, N., Doucet, C., et al. 2005, Icarus, 174, 90

Davidsson, B. J. R. 2001. Icarus, 149, 375

Davidsson, B. J. R., \& Gutiérrez, P. J. 2005, Icarus, in press

Davies, J. K., McBride, N., Ellison, S. L., Green, S. F., \& Ballantyne, D. R. 1998, Icarus, 134, 213

Davis, D. R., \& Farinella, P. 1997, Icarus, 125, 50

Duncan, M., Quinn, T., \& Tremaine, S. 1988, ApJ, 328, L69

Farnham, T. L. 2001, BAAS, 33, \#12.10

Fernández, J. A. 1980, MNRAS, 192, 481
Fornasier, S., Doressoundiram, A., Tozzi, G. P., et al. 2004, A\&A, 421, 353

Gutiérrez, P. J., Ortiz, J. L., Alexandrino, E., Roos-Serote, M., \& Doressoundiram, A. 2001, A\&A, 371, L1

Hainaut, O. R., Delahodde, C. E., Boehnhardt, H., et al. 2000, A\&A, 356,1076

Hainaut, O. R., \& Delsanti, A. C. 2002, A\&A, 389, 641

Housen, K. R. 2004, Lunar and Planetary Science, 35, 1826, A\&A, 389,641

Jewitt, D. C., \& Luu, J. X. 2001, AJ, 122, 2099

Lacerda, P., \& Luu, J. 2003, Icarus, 161, 174

Lagerkvist, C. I., Harris, A. W., \& Zappala, V. 1989, Asteroids II; Proceedings of the Conference, Tucson, AZ, Mar. 8-11, 1988 (University of Arizona Press), 1162

Lazzarin, M., Barucci, M. A., Boehnhardt, H., et al. 2003, AJ, 125, 1554

Lomb, N. R. 1976, Astroph. Space Sci., 39, 447

Margot, J. L., Nolan, M. C., Benner, L. A., et al. 2002, Science, 296, 1445

Noll, K. S., Stephens, D. C., Grundy, W. M., et al. 2002, AJ, 124, 3424

Ortiz, J. L., Baumont, S., Gutiérrez, P. J., \& Roos-Serote, M. 2002, A\&A, 388, 661

Ortiz, J. L., Gutiérrez, P. J., Casanova, V., \& Sota, A. 2003a, A\&A, 407, 1149

Ortiz, J. L., Gutiérrez, P. J., Sota, A., et al. 2003b, A\&A, 409, L13

Ortiz, J. L., Sota, A., Morero, R., et al. 2004, A\&A, 420, 383

Pan, M., \& Sari, R. 2005, Icarus, in press

Peixinho, N., Doressoundiram, A., \& Romon-Martin, J. 2002, New Astron., 7, 359

Peixinho, N., Boehnhardt, H., Belskaya, I., et al. 2004, Icarus, 170, 153

Press, W. H., Teukolsky, S. A., Vetterling, W. T., \& Flannery, B. P. 1992, in Numerical Recipes in Fortran: the art of scientific computing, 2nd edition (London: Cambridge Univ. Press), 569

Richarson, D. C., Bottke, W. F., \& Love, S. G. 1998, Icarus, 134, 47

Sekiguchi, T., Boehnhardt, H., Hainaut, O. R., \& Delahodde, C. E. 2002, A\&A, 385, 281

Shepppard, S., \& Jewitt, D. C. 2002, AJ, 124, 1757

Shepppard, S., \& Jewitt, D. C. 2003, Earth, Moon and Planets, 92, 207

Shepppard, S., \& Jewitt, D. C. 2004, AJ, 127, 3023

Sridhar, S., \& Tremaine, S. 1992, Icarus, 95, 86

Stern, S. A. 2002, AJ, 124, 2300

Tegler, S. C., Romanishin, W., Consolmagno, G. J., et al. 2005, Icarus, in press 